\title{
Hypothyroidism masquerading as cerebellar ataxia
}

\author{
Sakshi Gagneja ${ }^{1, *}$, Sourya Acharya ${ }^{2}$, Anuja Beralkar ${ }^{3}$, Ayush Somani $^{4}$ \\ ${ }^{1}$ Senior Resident, ${ }^{2}$ Professor, ${ }^{3,4} \mathrm{PG}$ Resident, Dept. of General Medicine, Jawaharlal Nehru Medical College-Datta Meghe \\ Institute of Medical Sciences, Wardha, Maharashtra, India
}

*Corresponding Author:

Email: simplysakshi05@gmail.com

\begin{abstract}
Cerebellar ataxia can be a rare possible presentation of autoimmune hypothyroidism. We report a case of a young 20 years old female, who presented with bilateral cerebellar ataxia without any other significant neurological features. In detail investigations, she was discovered to be a case of Hashimoto Thyroiditis with positive anti-thyroid peroxidase (TPOAb) antibody and low freeT4 and freeT3 levels. She improved dramatically with levothyroxine therapy and iv pulse methylprednisolone. Thus, hypothyroidism should be looked for as a reversible and treatable cause in all cases of bilateral cerebellar ataxia.
\end{abstract}

Keywords: Hypothyroidism, Autoimmune, Cerebellar ataxia, TPOAb.

\section{Introduction}

Cerebellar ataxia is well known to be caused by a long list of infections, demyelination, metabolic factors, vascular insults, nutritional deficiency, paraneoplastic syndromes and some toxins. Hypothyroidism has been described as one of the reversible causes of cerebellar ataxia, although the exact pathophysiology is not well known. ${ }^{1-5}$ Hashimoto's thyroiditis has been described as a chronic inflammation of the thyroid gland and the most common cause of hypothyroidism. ${ }^{6}$ The ataxia has been observed to improve by thyroid replacement therapy in many reported cases, so possibly it could be due to the physiological effects of thyroid hormone deficient state. ${ }^{7-9}$ However, in many cases, the prognosis of the ataxia has been bad even after the hormonal therapy. ${ }^{1,3,7}$ We report a case of a young lady presenting with subacute onset bilateral cerebellar ataxia, which on detailed work up, found to be caused by autoimmune hypothyroidism. She was treated with levothyroxine and corticosteroids, showed remarkable improvement.

\section{Case Report}

A 20 years old, normotensive, nondiabetic right handed lady presented gait ataxia of about two months duration. First of all, she noticed irregularity in her writing two months back then over a span of one month, she discovered that she was facing difficulty in buttoning up her clothes and she has developed smearing of face while eating. Rapidly in a month, she became grossly ataxic with a tendency to fall while walking. There was no history of any loss of consciousness, fever, convulsion, headache, visual complaints or any other neurological symptoms. She gave no history of sensory disturbances and her bowel $\&$ bladder function was normal. Besides this, she also had three months of secondary amenorrhea. Her other medical history was unremarkable. She is the second child of her parents with no history of any consanguinity. Her parents, one elder brother all are healthy without any history of neurological or endocrine disorders.

General physical examination was normal. Neurologically, higher mental functions were intact. A horizontal nystagmus was present on lateral gaze bilaterally, she showed marked dysmetria in both upper and lower limbs. She also had dysdiadochokinesia. She had normal muscle bulk with hypotonia and slight distal weakness $(4+/ 5)$ in all limbs. Deep tendon reflexes were normal except diminished at the ankles. Plantar reflexes were flexor bilaterally. Sensory examination revealed no abnormality. Her gait was broad based and reeling to either side. Rest of the examination was normal.

She was investigated thoroughly for major causes of sub acute bilateral cerebellar ataxia. MRI brain with screening of spine with contrast was done which was normal (Fig. 1). TSH was raised(31Uiu) and FT4(2ug/dl), FT3 (40ng/dl) were low, further she was tested for anti TPO Ab which was strongly positive. Her thyroid ultrasound revealed tiny colloid cysts. Her ESR was $85 \mathrm{~mm} /$ hour. Her hemoglobin was $10.5 \mathrm{gm} \%$ with HCT $31.2 \%$ and MCV 109, though her Vit B12 and folate was normal. Other routine liver function test and renal parameters including serum electrolytes were normal. Possible viral infections including HSV, CMV, HIV were ruled out by serology. VDRL, TPHA for syphilis was negative and vasculitic panel including ANA, cANCA, pANCA was also negative. Analysis of CSF for ADA, VDRL, Oligoclonal bands (OCB) \& IgG Index was also normal. NCS of crossed limbs revealed no abnormality. She investigated for paraneoplastic antibodies which were negative. She even tested negative for anti-gliadin antibody. 


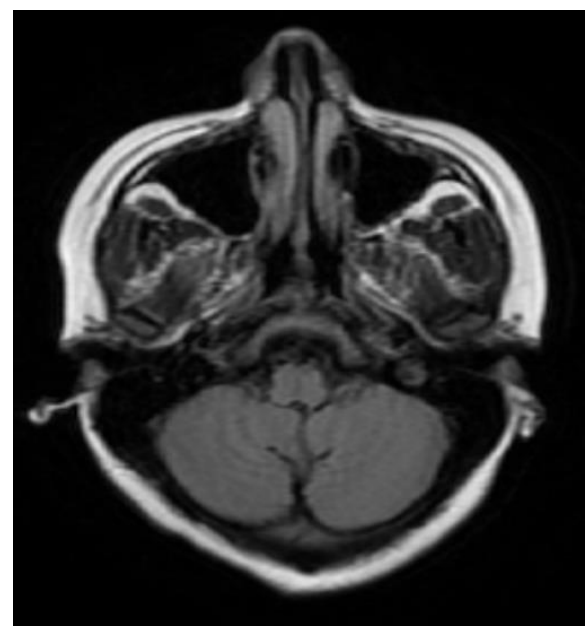

Fig. 1

Thus, after exhaustive investigations for cerebellar ataxia her hypothyroidism was the probable cause presenting in the form of cerebellar ataxia. She was started on thyroxin replacement therapy and iv methylprednisolone for three days to which she responded dramatically. Her symptoms improved in a due course of 2 months and her thyroid profile also stabilized. On long term follow up of one year, there was no recurrence of symptoms.

\section{Discussion}

Hypothyroidism is not new in literature as a reversible cause of cerebellar ataxia. However, the pathogenesis of cerebellar ataxia in hypothyroid state is not clear. It has been observed that treatment with Lthyroxin has reversed the ataxia in many patients thus there might be a possibility of endocrine associated dysfunction of the cerebellum. ${ }^{3,8}$ Pathophysiologically, it has been suggested that in hypothyroid state, there is a reduction of cardiac output, cerebral blood flow and metabolic insult by reduced oxygen and glucose consumption by cerebellar neurons which paramount to cerebellar ataxia. ${ }^{3,7,10}$ Hashimoto thyroiditis is a pathological condition in which there is an autoimmune destruction of thyroid gland resulting in functional deficiency. A case of encephalopathy has been reported in literature in association with Hashimoto's thyroiditis and which was steroid responsive indicating an autoimmune pathogenesis and also possibility of cerebral vasculitis. ${ }^{11-13}$ In one hypothesis, widespread autoimmune reactivity is seen in patients with Hashimoto's disease, so cerebellar degeneration may be mediated by unidentified circulating anti Purkinje cell antibody. ${ }^{14}$

Genetic evidences from studies of thyrotoxic periodic paralysis and spinocerebellar ataxia hint towards a possible association between autoimmune thyroid disease and Purkinje cell-type calcium channelopathy. ${ }^{15}$ Cerebellar dysfunction is also seen in congenital hypothyroidism. Studies demonstrate up to
40 percent of children and adolescents with congenital hypothyroidism show one or the other findings of cerebellar dysfunction, such as intention tremor, clumsy running, or unsteady stance. ${ }^{16}$ Animal studies have also supported the idea that hypothyroidism interferes with cerebellar development. ${ }^{17}$ Moreover, cerebellar ataxia resulting from congenital hypothyroidism has shown typical histological cerebellar changes but the prognosis is very poor not completely reversible with thyroxin therapy as we see in adult onset ataxia. ${ }^{18}$

Our case also suggests, the association of cerebellar ataxia with autoimmune hypothyroidism, supported by increased TPO-Ab. Studies also suggests a role of short-course of iv steroids in patients with the IgG4-related variant of Hashimoto Thyroiditis. ${ }^{19}$ We also treated our patient with steroids in view of the same.

\section{Conclusion}

We report a case of bilateral cerebellar ataxia in a young lady with autoimmune hypothyroidism with positive TPO-Ab. Hypothyroidism presenting as cerebellar ataxia is rare, but is one of the reversible causes. Profound signs of hypothyroidism may or may not be present. We highly recommend testing of thyroid function and antibodies in all cases presenting as acute or subacute onset cerebellar ataxia.

\section{References}

1. Barnard R, Campbell M, McDonald W. Pathological findings in a case of hypothyroidism with ataxia. $J$ Neurol Neurosurg Psychiatry 1971; 34:755-60.

2. Bonuccelli U, Nuti A, Monzani F, et al. Familial occurrence of hypothyroidism and cerebellar ataxia. Funct Neurol 1991;6:171-5.

3. Cremer GM, Goldstein NP, Paris J. Myxedema and ataxia. Neurol 1969;19:37-46.

4. Erkulvrawatr S. Gait ataxia in hypothyroidism. J Ky Med Assoc 1977;75:581-2.

5. Gentilini M, Palmieri M. Myxedematous cerebellar ataxia: description of a case. Rivista di Patologica Nervosae Mentale 1984;105:75-80.

6. Delemer B, Aubert JP, Nys P, Landron F, Bouee S. An observational study of the initial management of hypothyroidism in France: the ORCHIDEE study. Eur $J$ Endocrinol 2012;167:817-23.

7. Jellinek EH, Kelly RE. Cerebellar syndrome in myxoedema. Lancet 1960;2:225-7.

8. Söderbergh G. Encore un cas de myxoedeme avec symptomes cerebelleux. Rev Neurol (Paris) 1911;22:869.

9. Takayanagi K, Satoth A, Yoshimura T, et al. A case of myxoedema associated with cerebellar ataxia and various neurological findings. Nippon Naika Gakkai Zasshi 1982;71:995-9.

10. Tanaka M, Kawarabayashi T, Okamoto K, et al. Reduction of cerebral blood flow and metabolic rate of oxygen in a case of hypothyroidism presenting with cerebellar ataxia. Rinsho shinkeigaku 1987;27:1262-5.

11. Cohen L, Mouly S, Tassan P, et al. A woman with a relapsing psychosis who got better with prednisone. Lancet 1996;347:1228. 
12. Dinkar K, Sinha S, Taly A B, Bindu P S, Bharath R D. Steroid responsive encephalopathy in autoimmune thyroiditis: Clinical spectrum and MRI observations in three cases. Ann Ind Acad Neurol 2011;14:205-7.

13. Concetta M. Forchetti, G. Katsamakis, et al. Autoimmune thyroiditis and a rapidly progressive dementia: global hypoperfusion on SPECT scanning suggests a possible mechanism. Neurol 1997;49:623-6.

14. Chiu A, Sherman S. Clinical manifestations and differential diagnosis of hypothyroidism. In: Falk SA, ed. Thyroid disease: endocrinology, surgery, nuclear medicine, and radiotherapy.2nd ed. Philadelphia: Lippincott-Raven 1997:379-81.

15. Adams R, Victor M, Ropper A. Principles of neurology. 6thed. New York: McGraw-Hill 1997:1084-6.

16. Wiebel J. Cerebellar-ataxic syndrome in children and adoles- cents with hypothyroidism under treatment. Acta Paediatr Scand 1976;65(2):201-5.

17. Koibuchi N, Jingu H, Iwasaki T, Chin WW. Current perspectives on the role of thyroid hormone in growth and development of cer- ebellum. Cerebellum 2003;2(4):279-89.

18. Hammar CH, Regli F. Cerebellar ataxia due to hypothyroidism in adults (case report). Dtsch Med Wochenschr 1975;100(28):1504-6.

19. Watanabe T, Maruyama M, Ito T, Fujinaga Y, Ozaki Y, Maruyama M, et al. Clinical features of a new disease concept, IgG4-related thyroiditis. Scand J Rheumatol 2013;42:325-30. 